\title{
Non-Renegable Selective Acknowledgments (NR-SACKs) for SCTP
}

\author{
Preethi Natarajan ${ }^{1}$, Nasif Ekiz ${ }^{1}$, Ertugrul Yilmaz ${ }^{1}$, Paul D. Amer ${ }^{1}$, Janardhan Iyengar ${ }^{2}$, Randall \\ Stewart ${ }^{3}$ \\ ${ }^{1}$ CIS Dept., University of Delaware, \{nataraja,ekiz,yilmaz,amer\}@ cis.udel.edu, \\ ${ }^{2}$ Math \& Computer Science, Franklin \& Marshall College, jiyengar@fandm.edu \\ ${ }^{3}$ Cisco Systems, rrs@lakerest.net
}

\begin{abstract}
In both TCP and SCTP, selectively acked (SACKed) out-of-order data is implicitly renegable; that is, the receiver can later discard SACKed data. The possibility of reneging forces the transport sender to maintain copies of SACKed data in the send buffer until they are cumulatively acked. In this paper, we investigate the situation where all out-oforder data is non-renegable, such as when the data has been delivered to the application, or when the receiver simply never reneges. Using simulations, we show that SACKs result in inevitable send buffer wastage, which increases as frequency of loss events and loss recovery durations increase. We introduce a fundamentally new ack mechanism, Non-Renegable Selective Acknowledgments (NR-SACKs), for SCTP. Using NR-SACKs, an SCTP receiver can explicitly identify some or all out-of-order data as being non-renegable, allowing the sender to free up send buffer sooner than if the data were only SACKed. We compare and show that NR-SACKs enable efficient utilization of a transport sender's memory. We further investigate the effects of using NR-SACKs in Concurrent Multipath Transfer (CMT). CMT is an experimental SCTP extension that exploits multihoming for simultaneous data transfer over multiple paths [4]. Using simulations, we show that NR-SACKs not only reduce transport sender's memory requirements, but also improve throughput in CMT.
\end{abstract}

Index Terms-Network Protocols, Protocol Design and Analysis

\section{INTRODUCTION}

Reliable transport protocols such as TCP and SCTP (Stream Control Transmission Protocol) [RFC4960] employ two kinds of data acknowledgment mechanisms: (i) cumulative acks indicate data that has been received in-sequence, and (ii) selective acknowledgments (SACKs) indicate data that has been received out-of-order. In both TCP and SCTP, while cumulatively acked data is the receiver's responsibility,

\footnotetext{
- Prepared through collaborative participation in the Communication and Networks Consortium sponsored by the US Army Research Lab under Collaborative Tech Alliance Program, Coop Agreement DAAD19-01-20011. The US Gov't is authorized to reproduce and distribute reprints for Gov't purposes notwithstanding any copyright notation thereon.

- Supported by the University Research Program, Cisco Systems, Inc.
}

SACKed data is not, and SACK information is advisory [RFC3517, RFC4960]. While SACKs notify a sender about the reception of specific out-of-order TPDUs, the receiver is permitted to later discard the TDPUs. Discarding data that has been previously SACKed is known as reneging. Though reneging is a possibility, the conditions under which current transport layer and/or operating system implementations renege, and the frequency of these conditions occurring in practice (if any) are unknown and needs further investigation.

Data that has been delivered to the application, by definition, is non-renegable by the transport receiver. Unlike TCP which never delivers out-of-order data to the application, SCTP's multistreaming and unordered data delivery services result in out-of-order data being delivered to the application and thus become non-renegable. Interestingly, TCP and SCTP implementations can be configured such that the receiver is not allowed to and therefore never reneges on out-of-order data (details in Section II). In these configurations, even nondeliverable out-of-order data becomes non-renegable.

The current SACK mechanism in both TCP and SCTP does not differentiate between out-of-order data that "has been delivered to the application and/or is non-renegable" vs. data that "has not yet been delivered to the application and is renegable". In this work, we introduce a fundamentally new third acknowledgment mechanism called Non-Renegable Selective Acknowledgments (NR-SACKs) that enable a transport receiver to explicitly convey non-renegable information to the sender on some or all out-of-order TPDUs. While this work introduces NR-SACKs for SCTP, the NRSACKs idea can be applied to any reliable transport protocol that uses selective acknowledgments and/or permits delivery of out-of-order data.

In this work, we investigate the effect of SCTP's SACK mechanism in situations where out-of-order data is nonrenegable, and identify conditions under which SACKs affect performance for an SCTP sender. We further investigate the effects of NR-SACKs on Concurrent Multipath Transfer (CMT), an experimental extension to SCTP that exploits SCTP's multihoming feature for simultaneous transfer of new 
data over multiple paths (details in Section II) [4]. Section III introduces the NR-SACK chunk for SCTP, and briefly explains sender and receiver side NR-SACK processing details. Section IV elaborates evaluation preliminaries such as ns-2 simulation topologies, parameters for cross-traffic generation, and performance metrics. Section $\mathrm{V}$ analyzes SACK vs. NR-SACK results for both SCTP and CMT. Section VI concludes our work

\section{PROBLEM DESCRIPTION}

\section{A. Background}

The SCTP (or TCP) layer send buffer, or the sender-side socket buffer (Figure 1), consists of two kinds of data: (i) new application data waiting to be transmitted for the first time, and (ii) copies of data that have been transmitted at least once and are waiting to be cum-acked, a.k.a. the retransmission queue (rtxq). Data in the rtxq is the transport sender's responsibility until the receiver has guaranteed their delivery to the receiving application, and/or the receiver guarantees not to renege on the data.

In traditional in-order data delivery service, a receiver cumulatively acknowledges (cum-acks) the latest in-order data. Cum-acked data has either been delivered to the application or is ready for delivery. In either case, cum-acks are an explicit assurance that the receiver will not renege on the corresponding data. Upon receiving a cum-ack, the sender is no longer responsible, and removes the corresponding data from the rtxq. In the current SACK mechanism, cum-acks are the only means to convey non-renegable information; all selectively acknowledged (out-of-order) data are by default renegable.

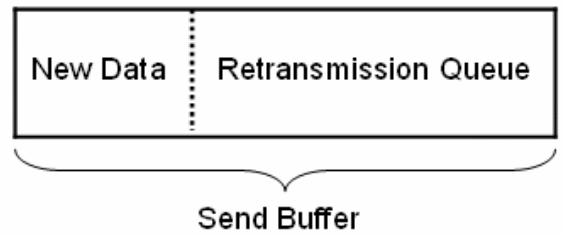

Figure 1: Transport Layer Send Buffer

SCTP's multistreaming service divides an end-to-end association (SCTP's term for a transport connection) into independent logical data streams. Data arriving in-sequence within a stream can be delivered to the receiving application even if the data is out-of-order relative to the association's overall flow of data. Also, data marked for unordered delivery can be delivered immediately upon reception, regardless of the data's position within the overall flow of data. Thus, SCTP's data delivery services result in situations where out-of-order data is delivered to the application, and is thus non-renegable.

Operating systems allow configuration of transport layer implementations such that out-of-order data is never reneged. For example, in FreeBSD, the net.inet.tcp.do_tcpdrain or net.inet.sctp.do_sctp_drain sysctl parameters can be configured to never revoke kernel memory allocated to TCP or SCTP out-of-order data [9]. Thus, out-of-order data can also be rendered non-renegable through simple user configuration.

In the following discussions, "non-renegable out-of-order data" refers to data for which the transport receiver takes full responsibility, and guarantees not to renege either because (i) the data has been delivered (or is deliverable) to the application, or (ii) the receiving system (OS and/or transport layer implementation) guarantees not to revoke the allocated memory until after the data is delivered to the application. With the current SACK mechanism, non-renegable out-oforder data is selectively acked, and is (wrongly) deemed renegable by the transport sender. Maintaining copies of nonrenegable data in the sender's retransmission queue is unnecessary.

\section{B. SCTP Unordered Data Transfer with SACKs}

We now discuss the effects of SACKs in transfers where all out-of-order is non-renegable. The discussion is applicable to any type of reliable data delivery service (in-order, partialorder, unordered) where all out-of-order data is non-renegable, but uses the simple unordered SCTP data transfer example shown in Figure 2.

In this example, the SCTP send buffer denoted by the rectangular box can hold a maximum of eight TPDUs. Each SCTP PDU is assigned a unique Transmission Sequence Number (TSN). The timeline slice shown in Figure 2 picks up the data transfer at a point when the sender's cwnd $\mathrm{C}=8$, allowing transmission of 8 TPDUs (arbitrarily numbered with TSNs 11-18). Note that when TSN 18 is transmitted, the retransmission queue grows to fill the entire send buffer.

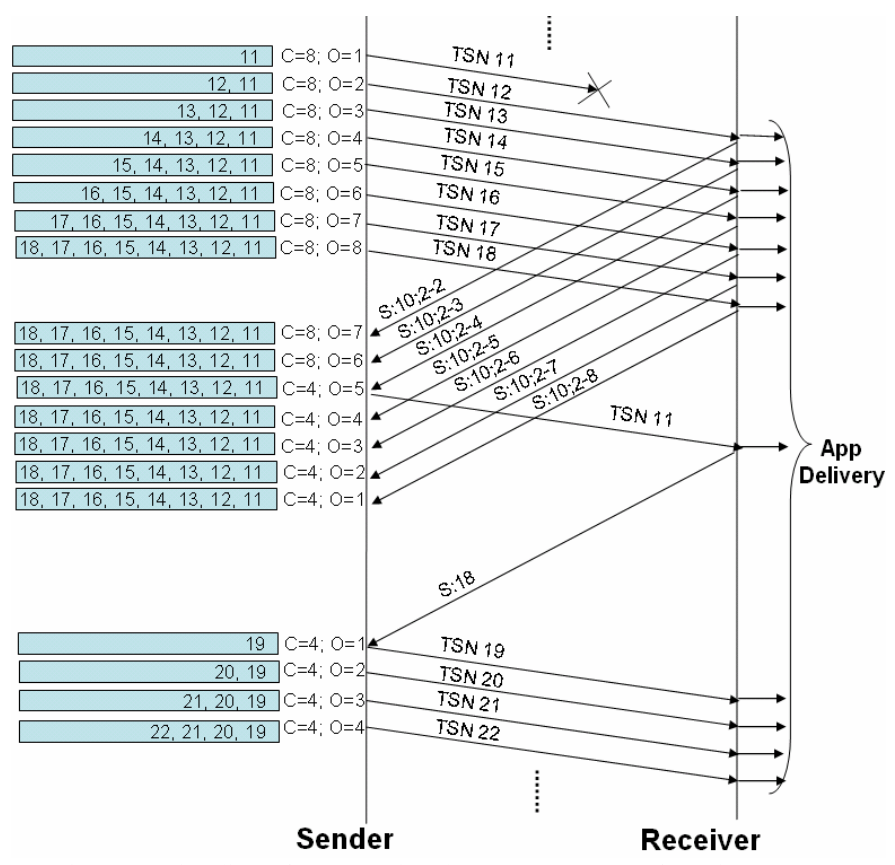

Figure 2: Timeline of an Unordered SCTP Data Transfer using SACKs

TSN 11 is presumed lost in the network. The other TSNs are received out-of-order and immediately SACKed by the SCTP receiver. The SACKs shown have the following format: (S)ACK:CumAckTSN;GapAckStart-GapAckEnd. Each Gap- 
ack start and Gap-ack end value is relative to the cum-ack value, and together they specify a block of received TSNs.

At the sender, the first SACK $(\mathrm{S}: 10 ; 2-2)$ is also a dupack and gap-acks TSN 12. Though data corresponding to TSN 12 has been delivered to the receiving application, the SACK does not convey the non-renegable nature of TSN 12, requiring the sender to continue being responsible for the TSN. Starting from the time that this SACK arrives at the sender, the copy of TSN 12 in the sender's rtxq is unnecessary. The gap-ack for TSN 12 reduces the amount of outstanding data $(\mathrm{O})$ to 7 TPDUs. Since $\mathrm{O}<\mathrm{C}$, the sender could in theory transmit new data, but in practice cannot do so since the completely filled send buffer blocks the sending application from writing new data into the transport layer. We call this situation send buffer blocking. Note that send buffer blocking prevents the sender from fully utilizing the cwnd.

The second and third dupacks (S:10;2-3, S:10;2-4) further increase the number of unnecessary TSNs in the rtxq. Send buffer blocking continues to prevent new data transmission. On receipt of the third dupack, the sender halves the cwnd $(\mathrm{C}=4)$, fast retransmits TSN 11, and enters fast recovery. Dupacks received during fast recovery further increase the amount of unnecessary data in the rtxq, prolonging inefficient rtxq usage. Note that though these dupacks reduce outstanding data $(\mathrm{O}<\mathrm{C})$, send buffer blocking prevents new data transmission.

The sender eventually exits fast recovery when the SACK for TSN 11's retransmission (S:18) arrives. The sender removes the unnecessary copies of TSNs 12-18 from the rtxq, and concludes the current instance of send buffer blocking. Since send buffer blocking prevented the sender from fully utilizing the cwnd before, the new cum ack (S:18) does not increase the cwnd [RFC4960]. The application writes new data into the newly available send buffer space and the sender now transmits TSNs 19-22.

The simple timeline demonstrates the following observations on transfers involving non-renegable out-of-order data:

- The unnecessary copies of non-renegable out-of-order data in the retransmission queue contribute to inefficient kernel memory usage. The amount of wasted memory is a function of flightsize (amount of data "in flight") during a loss event; larger flightsize increases the amount of wasted memory.

- When the retransmission queue grows to fill the entire send buffer, send buffer blocking ensues, which can degrade the transfer's throughput.

\section{Implications to Concurrent Multipath Transfer (CMT)}

A host is multihomed if it can be reached via multiple IP addresses, as is the case when the host has multiple network interfaces. SCTP supports transport layer multihoming for fault-tolerance purposes [RFC4960]. SCTP multihoming allows binding of an association to multiple IP addresses at each endpoint. An endpoint chooses a single destination address as the primary destination, which is used for all data traffic during normal transmission. SCTP also monitors the reachability of each destination address. Failure in reaching the primary destination results in failover, where an SCTP endpoint dynamically chooses an alternate destination to transmit the data.

Multiple active interfaces suggest the possibility of multiple independent end-to-end paths between the multihomed hosts. Concurrent Multipath Transfer (CMT) [4] is an experimental extension to SCTP that assumes multiple independent paths and exploits them for simultaneous transfer of new data between end hosts, and increases a network application's throughput. Similar to an SCTP sender, the CMT sender uses a single send buffer and rtxq for data transfer. However, the CMT sender's total flightsize is the sum of flightsizes on each path. Since the amount of kernel memory and the probability of send buffer blocking increase as the transport sender's flightsize increases (previous Section), we hypothesize that a CMT association is even more likely than an SCTP association to suffer from the inefficiencies of the existing SACK mechanism.

\section{NON-RENEGABLE SELECTIVE ACKNOWLEDGMENTS (NR- SACKS) FOR SCTP}

Non-Renegable Selective Acknowledgments (NR-SACKs) for SCTP [5] enables a receiver to explicitly convey nonrenegable information on out-of-order TPDUs. NR-SACKs provide the same information as SACKs for SCTP's congestion and flow control, and the sender is expected to process this information identical to SACK processing. In addition, NR-SACKs provide the option to report some or all of the out-of-order TPDUs as being non-renegable.

\section{A. NR-SACK Chunk Details}

The proposed NR-SACK chunk for SCTP is shown in Figure 3. Before sending/receiving NR-SACKs, the endpoints first negotiate NR-SACK usage during association establishment. An endpoint supporting the NR-SACK extension lists the NR-SACK chunk in the Supported Extensions Parameter carried in the INIT or INIT-ACK chunk [RFC5061]. During association establishment, if both endpoints support the NR-SACK extension, then each endpoint acknowledges received data with NR-SACK chunks instead of SACK chunks.

Since NR-SACKs extend SACK functionality, an NRSACK chunk has several fields identical to the SACK chunk: the Cumulative TSN Ack, the Advertised Receiver Window Credit (a_rwnd), Gap Ack Blocks, and Duplicate TSNs. These fields have identical semantics to the corresponding fields in the SACK chunk [RFC4960]. NR-SACKs also report nonrenegable out-of-order data chunks in the NR Gap Ack Blocks, a.k.a. "nr-gap-acks". Each NR Gap Ack Block acknowledges a continuous subsequence of non-renegable out-of-order data chunks. All data chunks with TSNs $\geq$ (Cumulative TSN Ack + NR Gap Ack Block Start) and $\leq$ (Cumulative TSN Ack + NR Gap Ack Block End) of each NR Gap Ack Block are reported as non-renegable. The Number of NR Gap Ack Blocks (M) 
field indicates the number of NR-Gap Ack Blocks included in the NR-SACK chunk.

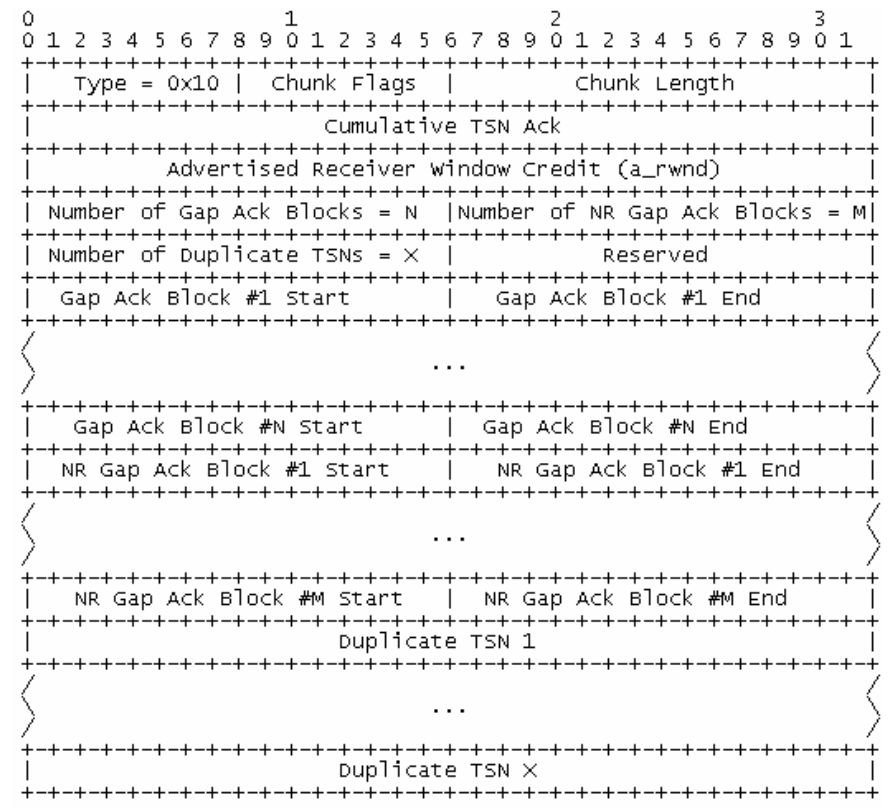

Figure 3: NR-SACK Chunk for SCTP

The third least significant bit in the Chunk Flags field is the (A) $l l$ bit. If the 'A' bit is set to ' 1 ', all out-of-order data blocks acknowledged in the NR-SACK chunk are non-renegable. The 'A' bit enables optimized sender/receiver processing and reduces the size of NR-SACK chunks when all out-of-order TPDUs at the receiver are non-renegable.

Note that each sequence of TSNs in an NR Gap Ack Block will be a subsequence of one of the Gap Ack Blocks, and there can be more than one NR Gap Ack Block per Gap Ack Block. Also, non-renegable information cannot be revoked. If a TSN is nr-gap-acked in any NR-SACK chunk, then all subsequent NR-SACKs gap-acking that TSN should also nr-gap-ack that TSN. Complete details of NR-SACK chunk can be found in [5].

\section{B. SCTP Unordered Data Transfer with NR-SACKs}

NR-SACKs provide an SCTP receiver with the option to convey non-renegable information on some or all out-of-order TPDUs. When a receiver guarantees not to renege an out-oforder data chunk and nr-gap-acks the chunk, the sender no longer needs to keep that particular data chunk in its rtxq, thus allowing the data sender to free up kernel memory sooner than if the data chunk were only gap-acked.

Figure 4 is analogous to Figure 2's example, this time using NR-SACKs. The sender and receiver are assumed to have negotiated the use of NR-SACKs during association establishment. As in the example of Figure 2, TSNs 11-18 are initially transmitted, and TSN 11 is presumed lost. For each TSN arriving out-of-order, the SCTP receiver transmits an NR-SACK chunk instead of SACK chunk. Since all out-oforder data are non-renegable in this example, every NR-SACK chunk has the ' $A$ ' bit set, and the nr-gap-acks report the list of
TSNs that are received out-of-order and non-renegable.

All NR-SACKs in Figure 4 have the following format: (N)R-SACK:CumAckTSN;NRGapAckStart-NRGapAckEnd. The first NR-SACK $(\mathrm{N}: 10 ; 2-2)$ is also a dupack. This NRSACK cum-acks TSN 10, and (nr-)gap-acks TSN 12. Once the data sender is informed that TSN 12 is non-renegable, the sender frees up the kernel memory allocated to TSN 12, allowing the application to write more data into the newly available send buffer space. Since TSN 12 is also gap-acked, the amount of outstanding data $(\mathrm{O})$ is reduced to 7, allowing the sender to transmit new data - TSN 19.

On receipt of the $2^{\text {nd }}$ and $3^{\text {rd }}$ dupacks that newly (nr-)gapack TSNs 13 and 14, the sender removes these TSNs from the rtxq. On receiving the second dupack, the sender transmits new data - TSN 20. On receipt of the third dupack, the sender halves the cwnd $(\mathrm{C}=4)$, fast retransmits TSN 11, and enters fast recovery. Dupacks received during fast recovery (nr-)gapack TSNs 15-20. The sender frees rtxq accordingly, and transmits new TSNs 21, 22 and 23. The sender exits fast recovery when the NR-SACK with new cum-ack (N:20) arrives. This new cum-ack increments $\mathrm{C}=5$, and decrements $\mathrm{O}=3$. The sender now transmits new TSNs 24 and 25 .

The explicit non-renegable information in NR-SACKs ensures that the rtxq contains only necessary data - TPDUs that are actually in flight or "received and renegable". Comparing Figures 2 and 4, we observe that NR-SACKs use the rtxq more efficiently.

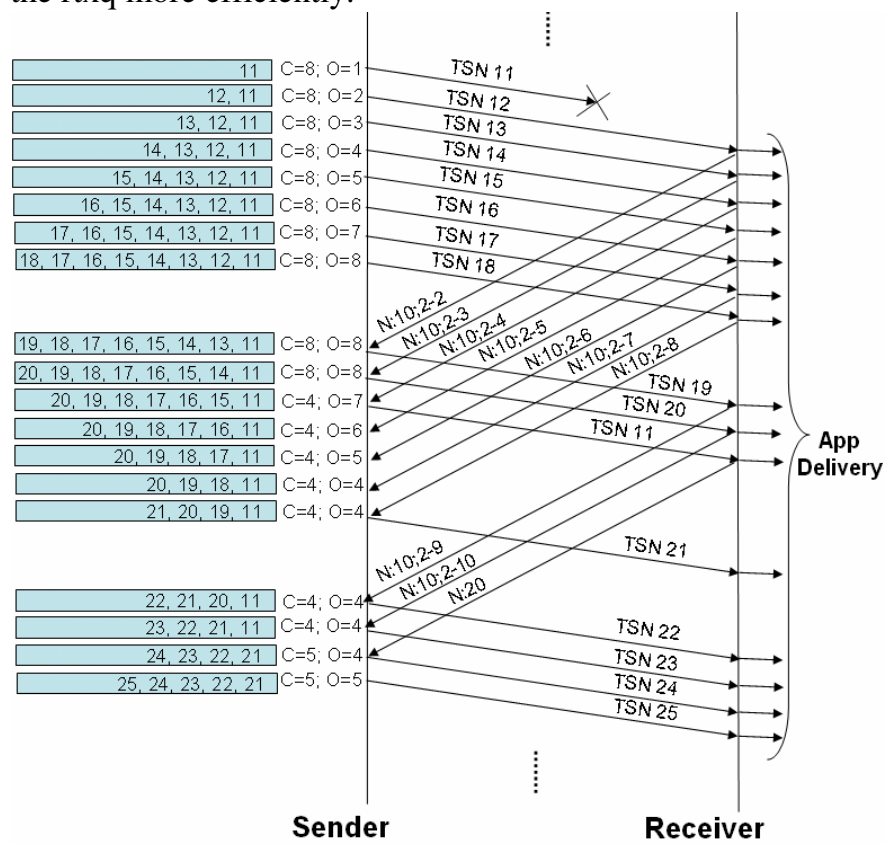

Figure 4: Timeline of an Unordered SCTP Data Transfer using NR-SACKs

\section{Evaluation PRELIMINARIES}

Ns-2 SCTP and CMT modules [??,3] were extended to support and process NR-SACK chunks. The simulation-based evaluations compare long-lived SCTP or CMT flows using SACKs vs. NR-SACKs under varying cross-traffic loads. This section discusses the experiment setup and other evaluation preliminaries in detail. 


\section{A. Simulation Setup}

Reference [1] recommends specific simulation setup and parameters for a realistic evaluation of TCP extensions and congestion control algorithms. These recommendations include network topologies, details of cross-traffic generation, and delay distributions mimicking patterns observed in the Internet. We adhere to these recommendations for a realistic evaluation of SACKs vs. NR-SACKs.
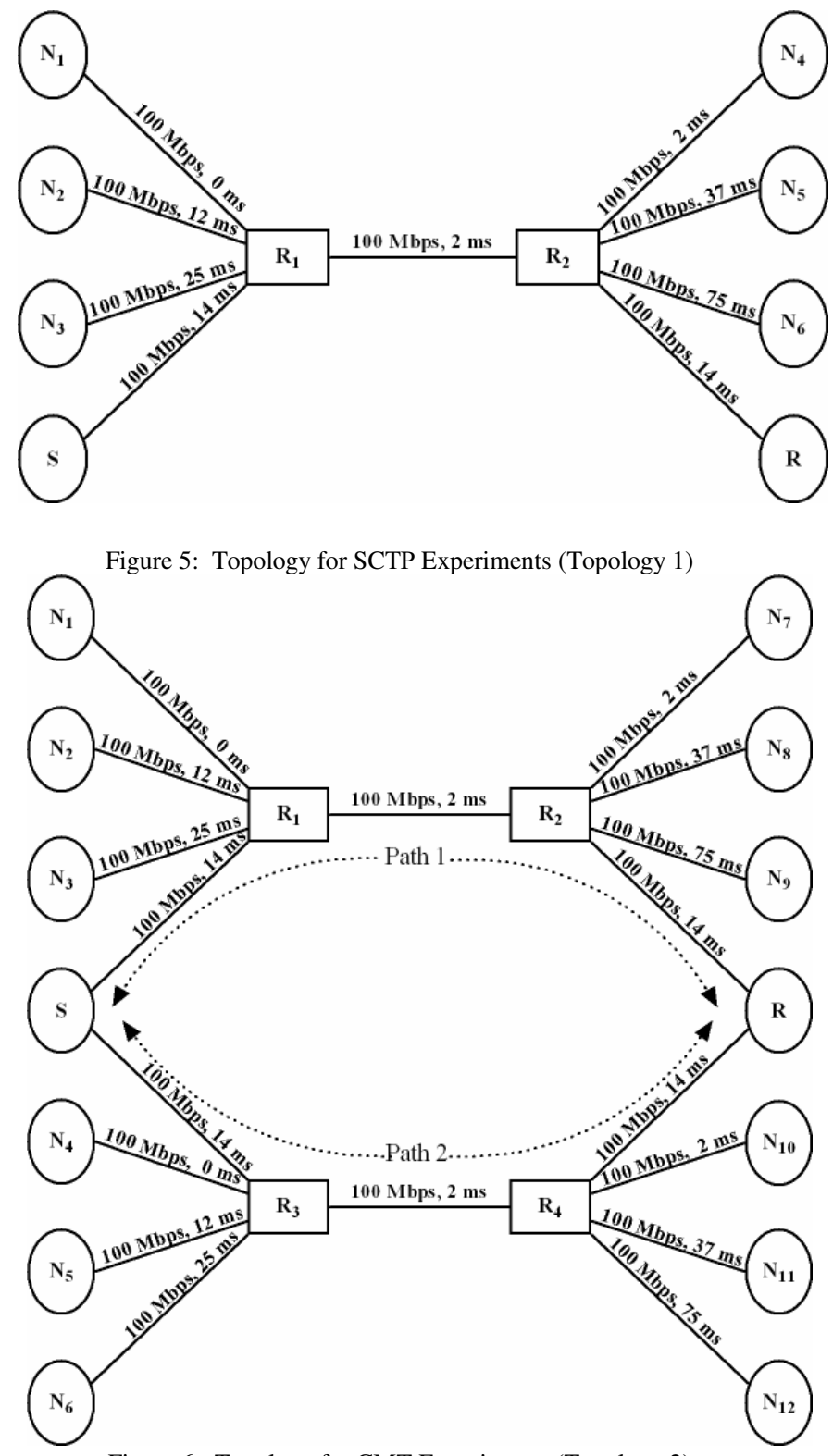

Figure 6: Topology for CMT Experiments (Topology 2)

The SCTP evaluations use the dumb-bell topology shown in Figure 5, which models the access link scenario specified in [1]. The central bottleneck link connects routers $\mathrm{R}_{1}$ (left) and $\mathrm{R}_{2}$ (right), has a $100 \mathrm{Mbps}$ capacity, and $2 \mathrm{~ms}$ one-way propagation delay. Both routers employ drop tail queuing and the queue size is set to the bandwidth-delay product of a $100 \mathrm{~ms}$ flow. Each router is connected to three cross-traffic generating edge nodes via $100 \mathrm{Mbps}$ edge links with the following propagation delays: $0 \mathrm{~ms}, 12 \mathrm{~ms}, 25 \mathrm{~ms}$ (left) and
$2 \mathrm{~ms}, 37 \mathrm{~ms}, 75 \mathrm{~ms}$ (right). Each left edge node generates crosstraffic destined to every right edge node and vice-versa. Thus, without considering queuing delays, the RTTs for cross-traffic flows sharing the bottleneck link range from $8 \mathrm{~ms}-204 \mathrm{~ms}$.

Reference [1] recommends application level cross-traffic generation over packet level generation, since, in the latter scenario, cross-traffic flows do not respond to the user/application/transport behavior of competing flows. Also, [1] proposes the use of Tmix [8] traffic generator. However, the recommended Tmix connection vectors were unavailable at the time of performing our evaluations. Therefore, we decided to employ existing ns-2 application level traffic generation tools, recommended by [7, ?]. Since our simulation setup uses application level cross-traffic, we believe that the general conclusions from our evaluations will hold for evaluations using the Tmix traffic generator.

Cross-traffic generated by three kinds of applications are considered: (i) non-greedy, responsive HTTP sessions generated by PackMime implementation [2], (ii) rate controlled, unresponsive video sessions over UDP, and (iii) greedy, responsive bulk file transfer sessions over TCP. We are unaware of existing measurement studies on the proportion of each kind of traffic observed in the Internet. Therefore the simulations assume a simple, yet reasonable rule for traffic mix proportion - more HTTP traffic than video or FTP traffic.

Each edge node runs a PackMime session to every edge node on the other side, and the amount of HTTP traffic generated is controlled via the PackMime rate parameter. Similarly, each edge node establishes video and FTP sessions to every edge node on the other side, and the number of video/FTP sources at each node impacts the amount of video/FTP traffic. To avoid synchronization issues, the PackMime, video, and FTP sessions start at randomly chosen times during the initial 5 seconds of the simulation. The default segment size for all TCP traffic results in 1500 byte IP PDUs; the segment size for $10 \%$ of the FTP flows is modified to result in 576 byte IP PDUs. Also, the PackMime request and response size distributions are seeded in every simulation run, resulting in a range of packet sizes at the bottleneck [1].

The bottleneck router load is measured as $(\mathrm{L})=$ (mean queue length $\div$ total queue size). Four packet-level load/congestion variations are considered: (i) Low $(\sim 15 \%$ load, $<0.1 \%$ loss), (ii) Mild ( $45 \%$ load, $1-2 \%$ loss), (iii) Medium ( $\sim 60 \%$ load, $3-4 \%$ loss), (iv) Heavy ( $\sim 5 \%$ load, 8 $9 \%$ loss).

Topology 1 (Figure 5) is used to evaluate SCTP flows. CMT evaluations are over the dual-dumbbell topology shown in Figure 6 (topology 2), with two independent bottleneck links between routers $R_{1}-R_{2}$ and $R_{3}-R_{4}$. Similar to topology 1 , each router in topology 2 is attached to 3 cross-traffic generating edge nodes, with similar bottleneck and edge link bandwidth/delay characteristics. In both topologies, nodes $\mathrm{S}$ and $\mathrm{R}$ are the SCTP or CMT sender and receiver, respectively. In topology 2, both $\mathrm{S}$ and $\mathrm{R}$ are multihomed, and the CMT sender uses the two independent paths (paths 1 and 2) for 
simultaneous data transfer. In both topologies, $\mathrm{S}$ and $\mathrm{R}$ are connected to the bottleneck routers via 100Mbps duplex edge links, with $14 \mathrm{~ms}$ one-way delay. Thus the one-way propagation delay experienced by the SCTP or the CMT flow corresponds to $30 \mathrm{~ms}$, approximately the US coast-to-coast propagation delay [6].

In both topologies, the bottleneck links experience bidirectional cross-traffic; the cross-traffic load is similar on both forward and reverse directions. In topology 1, the crosstraffic load varies from low to heavy. For CMT evaluations using topology 2 , the bottlenecks experience asymmetric path loads; path 1 cross-traffic load varies from low to heavy, while path 2 experiences low load.

The SCTP or CMT flow initiates an unordered data transfer 18-20 seconds after the simulation begins such that, all data received out-of-order at $\mathrm{R}$ is deliverable, and thus, nonrenegable. Trace collection begins after a 20 second warm-up period from the start of SCTP or CMT traffic, and ends when the simulation completes after 70 seconds. The CMT sender uses the recommended RTX-SSTHRESH retransmission policy, i.e., retransmissions are sent on the path with highest ssthresh [4].

\section{B. Metric: Retransmission Queue Utilization}

In transfers using SACKs, the rtxq consists of two kinds of data (Figure 2): (i) necessary data - data that is either "in flight" and has not yet reached receiver's transport layer, or data that has been received but is renegable by the transport receiver, and (ii) unnecessary data - data that is received out of order and is non-renegable. The rtxq is most efficiently utilized when all data in the rtxq are necessary. As the fraction of unnecessary data increases, the rtxq is less efficiently utilized.

The transport sender modifies the $\mathrm{rtxq}$ as and when SACKs/NR-SACKs arrive. The rtxq size varies during the course of a file transfer, but can never exceed the send buffer size. For time duration $t_{i}$ in the transfer, let

$r_{i}=$ size of rtxq, and

$k_{i}=$ amount of necessary data in the rtxq.

During $t_{i}$, only $k_{i} \div r_{i}$ of the rtxq is efficiently utilized, and the efficiency changes whenever $k_{i}$ or $r_{i}$ changes.

Let $\frac{k_{0}}{r_{0}}, \frac{k_{1}}{r_{1}}, \ldots \frac{k_{n}}{r_{n}}$ be the efficient rtxq utilization values during time durations $t_{0}, t_{1}, \ldots t_{n}\left(\sum t_{i}=T\right)$, respectively. The time weighted efficient rtxq utilization averaged over $T$ is calculated as RtxQ_Util $=\left(\sum t_{i} \times \frac{k_{i}}{r_{i}}\right) \div T$. To measure rtxq utilization, the ns-2 SCTP or CMT sender tracks $k_{i}, r_{i}$ and $t_{i}$ until association shutdown. Let,

$\mathrm{W}=$ time when trace collection begins after the initial warm-up time, and

$\mathrm{E}=$ simulation end time.

In the following discussions, the time weighted efficient rtxq utilization averaged over the entire trace collection time, i.e., $T=(E-W)$, is referred to as $R t x Q \_U t i l$.

In an unordered transfer using NR-SACKs, all out-of-order data will be nr-gap-acked and the rtxq should contain only necessary data. Therefore, we expect an SCTP or CMT flow using NR-SACKs to most efficiently utilize the rtxq $($ RtxQ_Util $=1)$ under all circumstances.

\section{Retransmission Queue Utilization during Loss Recovery}

Typically, in SCTP transfers, data is always received inorder during no losses, unless the intermediate routers reorder packets. Consequently, during no losses, SCTP flows employing either SACKs or NR-SACKs utilize the rtxq most efficiently, and both their RtxQ_Util values equal unity. The two acknowledgment mechanisms differ in rtxq usage only when data is received out-of-order, which ensues when an SCTP flow suffers packet losses. Specifically, in SCTP, the duration of NR-SACKs' impact on the rtxq is limited to loss recovery periods. To evaluate the impact of the two ack schemes during loss recovery periods, the ns-2 SCTP sender timestamps every entry/exit to/from loss recovery. Since none of the routers reorder packets in our simulations, the SCTP sender uses the following naive rule - sender enters loss recovery on the receipt of SACKs/NR-SACKs with at least one gap-ack block, and exits loss recovery on the receipt of SACKs/NR-SACKs with a new cum-ack and zero gap-acks. We found that this simple rule resulted in a good approximation of the actual loss recovery periods. In addition to RtxQ_Util, an SCTP sender also tracked RtxQ_Util_L, which corresponds to rtxq utilization averaged over only the loss recovery durations of trace collection.

Depending on the paths' bandwidth/delay characteristics, a CMT association experiences data reordering even under no loss conditions [4]. Data transmitted on the shorter RTT path will be received out-of-order w.r.t. data transmitted on other path(s). Therefore, the naïve rule mentioned above cannot be employed to estimate entry/exit of CMT sender's loss recovery. Therefore, the CMT sender tracked only RtxQ_Util.

\section{RESULTS}

For each type of sender (SCTP or CMT), different send buffer sizes imposing varying levels of memory constraints are considered: $32 \mathrm{~K}, 64 \mathrm{~K}$ and INF (infinite or unlimited space) for SCTP, and $128 \mathrm{~K}, 256 \mathrm{~K}$ and INF for CMT. The results are averaged over 30 runs, and plotted with $95 \%$ confidence intervals. In the following discussions, SCTP transfers using SACKs or NR-SACKs are referred to as SCTP-SACKs and SCTP-NR-SACKs, respectively. Similarly, CMT using SACKs or NR-SACKs are referred to as CMT-SACKs and CMT-NR-SACKs.

\section{A. Retransmission Queue Utilization}

As the end-to-end path gets more congested, SCTP-SACKs' RtxQ_Util_L remains fairly consistent $\sim 0.5$ (Figure 7), while the RtxQ_Util decreases (Figure 8). The RtxQ_Util_L values indicate that irrespective of path loss rate, SCTP-SACKs 
efficiently utilize only $\sim 50 \%$ of rtxq during loss recovery; $\sim 50 \%$ of rtxq is wasted buffering unnecessary data.

At lower congestion levels (lower cross-traffic), the frequency of loss events and the fraction of transfer time spent in loss recovery are smaller, resulting in negligible rtxq wastage during the entire trace collection period (RtxQ_Util). As loss recoveries become more frequent, SCTP-SACKs' inefficient rtxq utilization during loss recovery lowers the corresponding RtxQ_Util values. The simulation results show that SCTP-SACKs waste on average $20 \%$ of the rtxq during moderate congestion and $\sim 30 \%$ during heavy congestion conditions. The amount of wasted kernel memory increases as the number of transport connections increase, and can be significant at a server handling large numbers of concurrent connections, such as a web server.

By definition of the RtxQ_Util metric, NR-SACKs are expected to utilize the rtxq most efficiently, even during loss recovery periods (Section IVB). The simulation results confirm this hypothesis. RtxQ_Util values for both SCTP-NRSACKs and CMT-NR-SACKs are unity.

In CMT evaluations, path 2 experiences low traffic load, while path 1's traffic load varies from low to heavy (Figure 6). Recall that a CMT sender transmits data concurrently on both paths. Asymmetric path congestion levels aggravate data reordering in CMT. As path 1 congestion level increases, TPDU losses on the higher congested path 1 cause data transmitted on the lower congested path 2 to arrive out-oforder at the receiver. CMT congestion control is designed such that losses on path 1 do not affect the cwnd/flightsize on path 2 [4]. While losses on path 1 are being recovered, sender continues data transmission on path 2 , increasing the amount of non-renegable out-of-order data in the rtxq. As the paths become increasingly asymmetric in their congestion levels, the amount of non-renegable out-of-order data in the rtxq increases, and brings down CMT-SACKs' RtxQ_Util values (Figure 9).

Increasing the send buffer/rtxq space improves SCTPSACKs' or CMT-SACKs' kernel memory (rtxq) utilization only to a certain degree. In Figures 8 and 9 RtxQ_Util for INF send buffer is essentially the upper bound on how efficient SCTP or CMT employing SACKs utilizes rtxq. Therefore, we conclude that TPDU reordering results in inevitable rtxq wastage in transfers using SACKs. The amount of wasted memory increases as TPDU reordering and loss recovery durations increase. Also, smaller send buffer sizes further degrade RtxQ_Util_L and RtxQ_Util values. This degradation is more pronounced in CMT (Figure 9). Further investigations reveal this effect to be due to send buffer blocking, discussed next.

\section{B. Send Buffer Blocking in CMT}

When the rtxq grows to fill the entire send buffer, send buffer blocking ensues, preventing the application from writing new data into the transport layer (Section IIA). In both SCTP and CMT, send buffer blocking increases as the send buffer is more constrained (decreases). In addition, CMT employs multiple paths for data transfer, increasing a sender's total flightsize in comparison to SCTP. Therefore, we hypothesized that CMT would suffer more send buffer blocking than SCTP (Section IIC). Indeed, in the simulations, CMT suffered significant send buffer blocking even for $128 \mathrm{~K}$ and $256 \mathrm{~K}$ send buffer sizes. In this section, we focus on the effects of send buffer blocking in CMT.

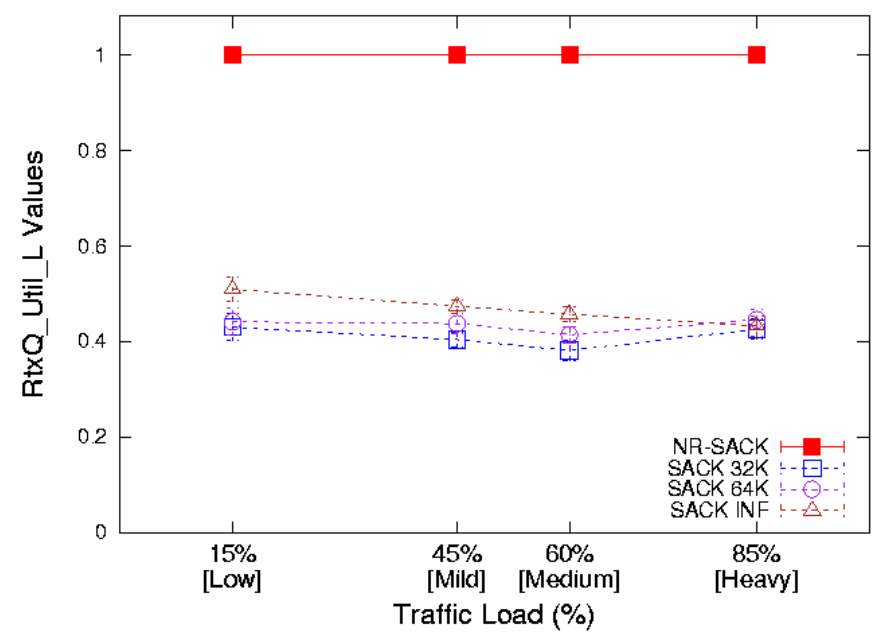

Figure 7: Rtxq Utilization during Loss Recovery in SCTP

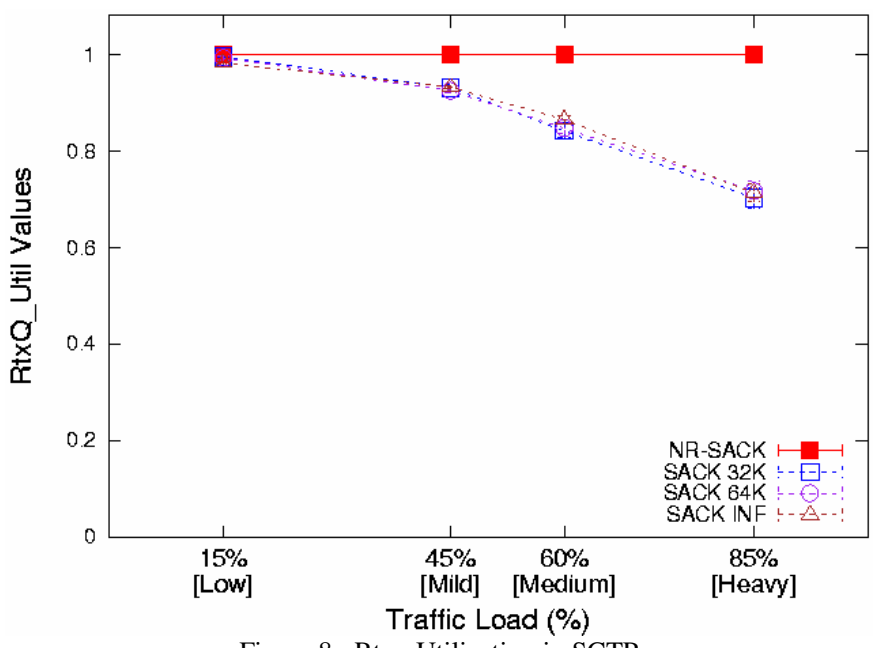

Figure 8: Rtxq Utilization in SCTP

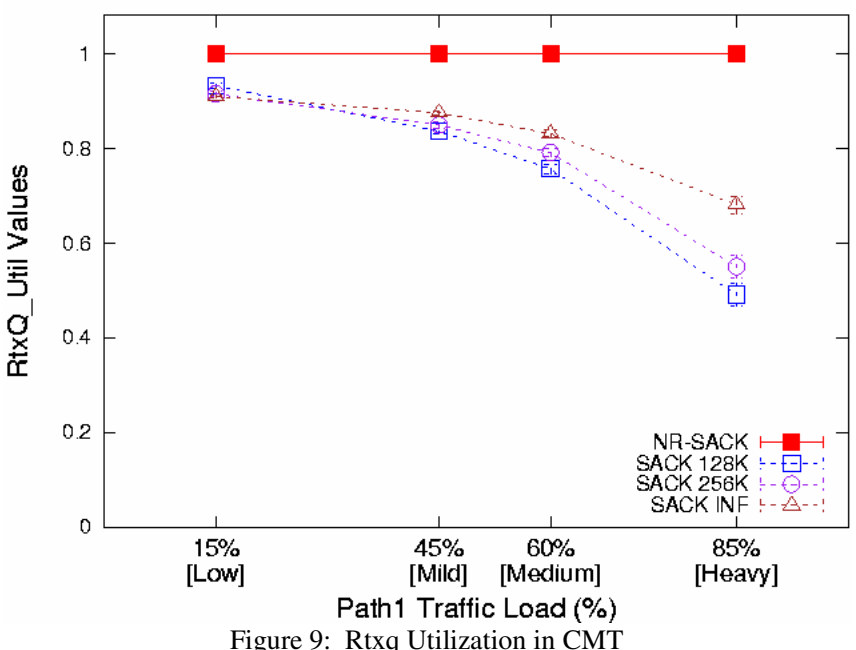




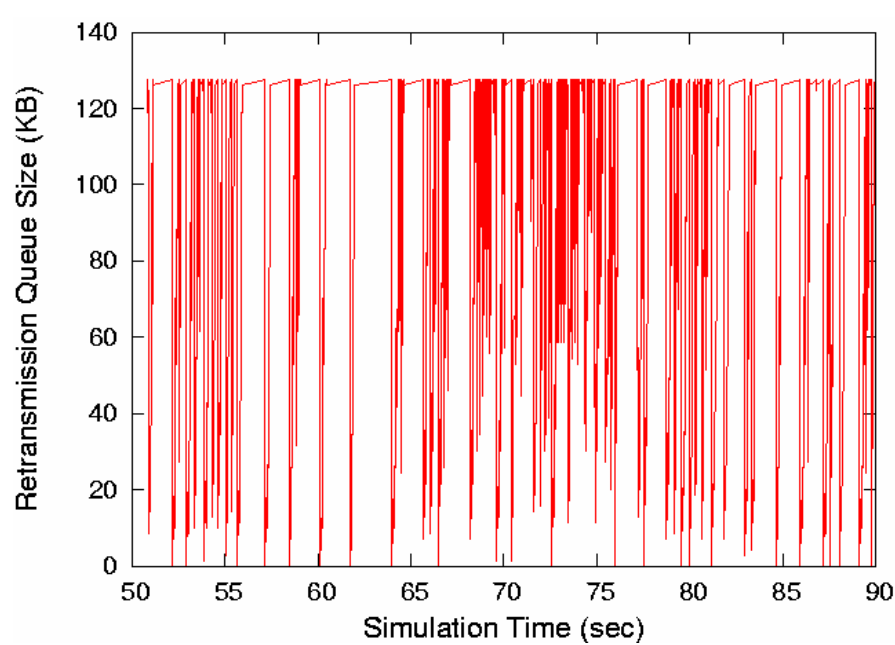

Figure 10: Retransmission Queue Evolution in CMT-SACKs

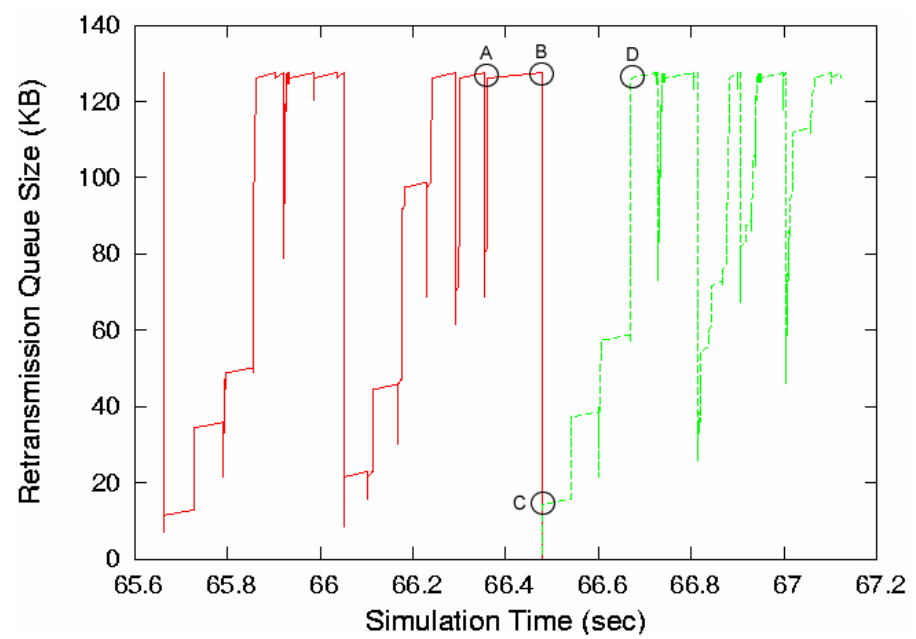

Figure 12: Retransmission Queue Evolution in CMT-SACKs (1.5 sec)

CMT using either acknowledgment scheme suffers from send buffer blocking for $128 \mathrm{~K}$ and $256 \mathrm{~K}$ buffer sizes. In CMT-SACKs, send buffer blocking continues until cum-ack point moves forward, i.e., until loss recovery ends. As path 1 congestion level increases, timeout recoveries become more frequent, causing longer loss recovery durations. Therefore, as congestion increases, the CMT-SACKs sender is blocked for larger fractions of transfer time. On the other hand, send buffer blocking in CMT-NR-SACKs is unaffected by the congestion level on path 1. As and when NR-SACKs arrive (on path 2), the CMT-NR-SACK sender removes nr-gap-acked data from the rtxq, allowing more data transmission.

CMT-SACKs' longer send buffer blocking durations adversely impact performance as discussed below.

\section{1) Ineffective Use of Send Buffer Space}

Send buffer blocking limits rtxq growth and reduces throughput. The impact on throughput is minimized when the available send buffer space is utilized as much as possible.

Figures 10 and 11 illustrate CMT sender's rtxq evolution over 40 seconds of a transfer using SACKs and NR-SACKs, respectively. The figures show that both CMT-SACKs and CMT-NR-SACKs suffer from send buffer blocking - the maximum rtxq size in the figures corresponds to $100 \%$ of send

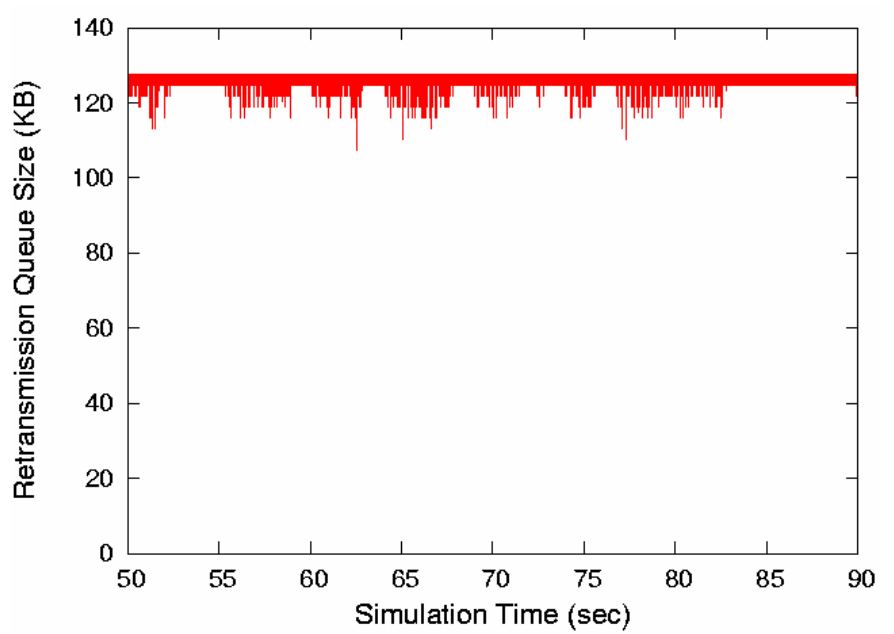

Figure 11: Retransmission Queue Evolution in CMT-NR-SACKs

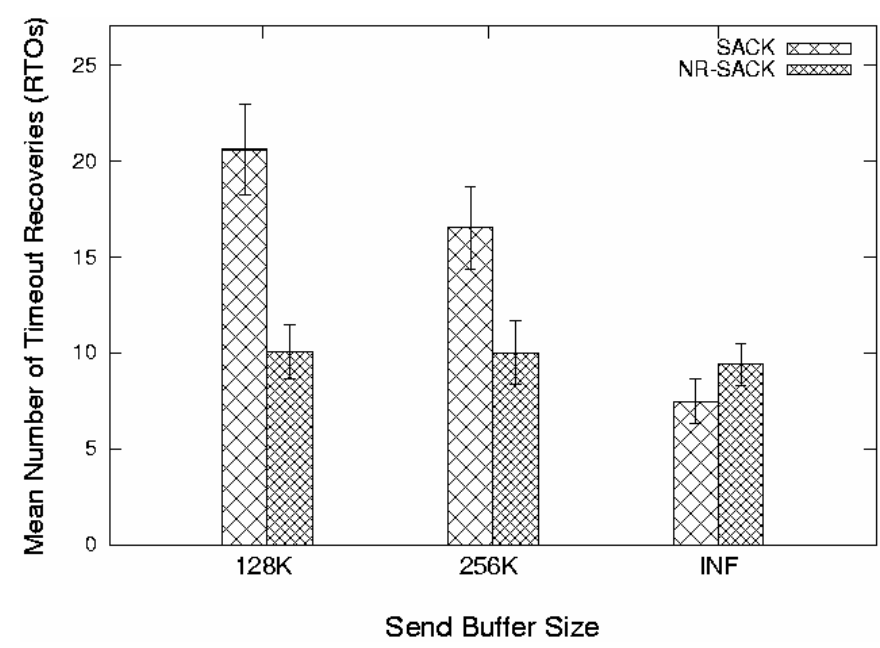

Figure 13: Mean Timeout Recoveries under Heavy Traffic Load in CMT

buffer (128K).However, the rtxq evolution in CMT-SACKs (Figure 10) exhibits more variance - reaches the maximum and drops to 0 multiple times, while CMT-NR-SACKs' rtxq size is closer to $128 \mathrm{~K}$ most of the time (Figure 11).

Figure 12 is a zoom of CMT-SACKs' rtxq evolution over an arbitrary 1.5 second period. At point A (time 66.36sec), rtxq size hits the maximum, and the sender is blocked from transmitting any more data. Subsequent SACKs reduce the amount of outstanding data, but send buffer blocking prevents the sender from clocking out new data. At time $66.42 \mathrm{sec}$, path 1 's retransmission timer expires; the sender detects loss, and retransmits TSN 20369 on path 2. At time 66.48sec (point B), sender receives a SACK with a new cum-ack $(\mathrm{TSN}=20457)$ and completely clears rtxq contents, ending the current instance of send buffer blocking. The sender immediately transmits new data on both paths, and the rtxq evolution after the new cum-ack (TSN=20457) is shown by the (green) dashed line. The cwnd on path 1 allows transmission of 2 MTU sized TPDUs (TSNs 20458 and 20459). The cwnd on path 2 is 127162 bytes, but the Maxburst parameter [RFC4960] limits the sender to transmit only 4 MTU sized TPDUs - TSNs 20460-20463. Once the sender transmits data on both paths, rtxq size increases to $\sim 8.6 \mathrm{~K}$, shown by point $\mathrm{C}$. 
Subsequent SACKs allow more data transmission and at point $\mathrm{D}$ the sender's rtxq reaches the maximum causing the next instance of send buffer blocking.

Though CMT-NR-SACKs (Figure 11) also incurs send buffer blocking, nr-gap-acks free up rtxq space allowing the sender to steadily clock out more data. A constrained send buffer is better utilized, and the transmission is less bursty with NR-SACKs than SACKs. The improved send buffer use contributes to throughput improvements (discussed later).

2) Retransmission Queue Utilization

In Figure 9, CMT-SACKs' RtxQ_Util worsens as send buffer blocking increases (send buffer size decreases). As discussed earlier, in CMT-SACKs, send buffer blocking prevents new data transmission until loss recovery. Lack of new data transmission resulted in fewer and sometimes insufficient acks to trigger fast retransmits. Consequently, blocked CMT-SACKs experienced more timeout recoveries (RTOs) at heavy traffic loads than non-blocked CMT-SACKs (Figure 13). As the send buffer is more constrained, the average number of RTOs increase, and the fraction of transfer time spent in loss recovery increases. Longer loss recovery durations increase the duration of inefficient rtxq utilization, and bring down blocked CMT-SACKs' RtxQ_Util values compared to non-blocked (INF) CMT-SACKs' RtxQ_Util.

On the other hand, CMT-NR-SACKs steadily clock out data, and do not incur excessive RTOs during send buffer blocking. CMT-NR-SACKs' mean number of RTOs for 128K and $256 \mathrm{~K}$ buffer sizes are similar to the INF case (Figure 13). To conclude, send buffer blocking worsens CMT-SACKs' rtxq utilization. Blocked CMT-SACKs' inefficient send buffer usage increases the number of timeout recoveries, and degrades throughput when compared to CMT-NR-SACKs.

\section{3) Throughput}

When the send buffer never limits rtxq growth (INF send buffer size), both CMT-SACKs and CMT-NR-SACKs experience no send buffer blocking, and perform similarly (Figure 14). However, CMT-SACKs achieve the same throughput as CMT-NR-SACKs at the cost of larger rtxq sizes.

Using terminology defined in Section IVB, the average rtxq size, Rtx $Q$ over the entire trace collection period $(T)$ is calculated as, $\operatorname{Rtx} Q=\left(\sum t_{i} \times r_{i}\right) \div T$. Figure 15 plots CMT-SACKs vs. CMT-NR-SACKs Rtx $Q$ for the INF case. As path 1 cross-traffic load increases, the bandwidth available for the CMT flow decreases, and CMT-NR-SACKs' RtxQ decreases (Figure 15). Similarly, CMT-SACKs' RtxQ decreases as traffic load increases from low to mild. However, a different factor dominates and increases CMT-SACKs' Rtx $Q$ during medium and heavy traffic conditions. Note that rtxq growth is never constrained in the INF case, enabling the CMT sender to transmit as much data as possible on path 2 while recovering from losses on path 1 . At medium and heavy crosstraffic loads, loss recovery durations increase due to increased timeout recoveries, and the CMT-SACKs sender transmits more data on path 2 compared to mild traffic conditions. This factor increases CMT-SACKs' RtxQ during medium and heavy traffic conditions.

Going back to Figure 14, when the send buffer size limits rtxq growth, CMT-NR-SACKs' efficient rtxq utilization alleviates send buffer blocking, and CMT-NR-SACKs perform better than CMT-SACKs. The throughput improvements in CMT-NR-SACKs increase as conditions that aggravate send buffer blocking increases. I.e., NR-SACKs improve throughput more as send buffer becomes more constrained and/or when the paths become more asymmetric in the congestion levels. Alternately, CMT-NR-SACKs achieve similar throughput as CMT-SACKs using smaller send buffer sizes. For example, during mild, medium and heavy path 1 cross-traffic load, CMT-NR-SACKs with $128 \mathrm{~K}$ send buffer performs similar or better than CMT-SACKs with 256K send buffer. Also, CMT-NR-SACKs with 256K send buffer performs similar to CMT-SACKs with larger (unconstrained) send buffer.

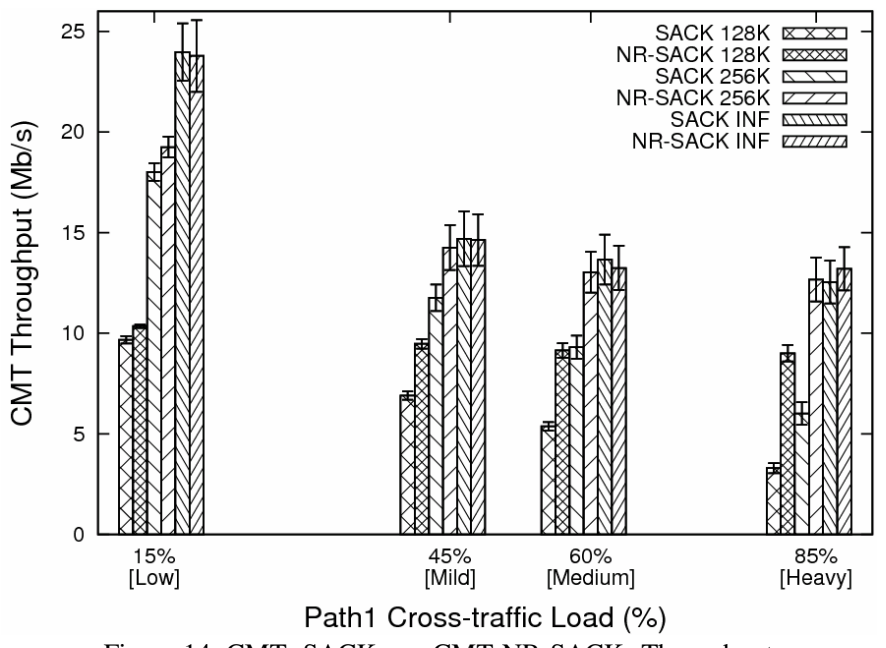

Figure 14: CMT- SACKs vs. CMT-NR-SACKs Throughput

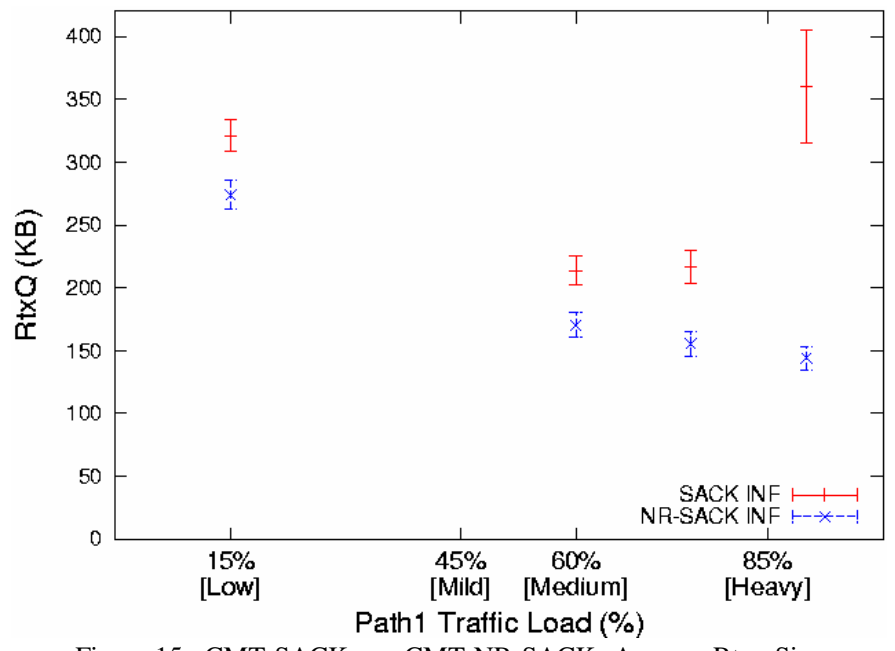

Figure 15: CMT-SACKs vs. CMT-NR-SACKs Average Rtxq Size

\section{CONCLUSION \& FUTURE WORK}

This work investigated the effect of existing SACK mechanism when data received out-of-order is non-renegable. We conclude that SACKs cause inevitable sender memory 
wastage, which worsens as data reordering and loss recovery durations increase. We proposed a new ack mechanism, NonRenegable Selective Acknowledgments for SCTP, which provides the transport receiver with the option to convey nonrenegable information on some or all out-of-order data.

A transfer employing NR-SACKs never performs worse than a transfer using SACKs. When out-of-order data is nonrenegable NR-SACKs perform better than SACKs. Simulations confirmed that in both SCTP and CMT, NRSACKs utilize send buffer and rtxq space most efficiently. Send buffer blocking in CMT with SACKs adversely impact end-to-end performance, while efficient send buffer use in CMT with NR-SACKs alleviates send buffer blocking. Therefore, NR-SACKs not only reduce sender's memory requirements, but also improve throughput in CMT. We are in the process of implementing NR-SACKs in FreeBSD and NRSACKs are being pursued as an experimental extension to SCTP in the IETF [5].

We plan to investigate the impact of asymmetric path delays in CMT with SACKs. Asymmetric path delays can aggravate send buffer blocking in CMT with SACKs, and we expect NRSACKs to alleviate this blocking.

\section{ACKNOWLEDGMENTS}

The authors acknowledge the valuable comments and suggestions from Dr. Philip Conrad at the University of California Santa Barbara, Jonathan Leighton and Joseph Szymanski at the University of Delaware's Protocol Engineering Lab.

\section{DISCLAIMER}

The views and conclusions contained in this document are those of the authors and should not be interpreted as representing the official policies, either expressed or implied, of the Army Research Laboratory or the U.S. Government

\section{REFERENCES}

[1] L. Andrew, C Marcondes, S. Floyd, L. Dunn, R. Guillier, W. Gang, L. Eggert, S. Ha, I. Rhee. Towards a Common TCP Evaluation Suite. In PFLDnet 2008, March 2008.

[2] J. Cao, W.S. Cleveland, Y. Gao, K. Jeffay, F.D. Smith, M.C, Weigle, "Stochastic Models for Generating Synthetic HTTP Source Traffic", INFOCOM, March 2004.

[3] N. Ekiz, P. Natarajan, J. Iyengar, A. Caro, "ns-2 SCTP Module," Version 3.7, September 2007. pel.cis.udel.edu.

[4] J. Iyengar, P. Amer, R. Stewart, "Concurrent Multipath Transfer using SCTP Multihoming over Independent End-to-end Paths," IEEE/ACM Transactions on Networking, October 2006, 14(5), pp 951-964.

[5] P. Natarajan, P. Amer, E. Yilmaz, R. Stewart, J. Iyengar, "NonRenegable Selective Acknowledgments (NR-SACKs) for SCTP," Internet Draft, draft-natarajan-tsvwg-sctp-nrsack (work in progress).

[6] S. Shakkottai, R. Srikant, A. Broido, K. Claffy, "The RTT distribution of TCP Flows in the Internet and its Impact on TCP-based flow control," TR, CAIDA, February, 2004.

[7] G. Wang, Y. Xia, D. Harrison, "An NS2 TCP Evaluation Tool," Internet Draft, April 2007.

[8] M. C. Weigle, P. Adurthi, F. Hern'andez-Campos, K. Jeffay, and F. D. Smith, "Tmix: a Tool for Generating Realistic TCP Application Workloads in ns-2," SIGCOMM Computer Communication Review (CCR), vol. 36, no. 3, pp. 65-76, 2006.
[9] FreeBSD TCP and SCTP Implementation, URL: http://www.freebsd.org/cgi/cvsweb.cgi/src/sys/netinet/\#dirlist.

[10] J. Iyengar, P. Amer, R. Stewart, "Performance implications of a bounded receive buffer in concurrent multipath transfer," Computer Communications, February 2007, 30(4), pp 818-829. 